\title{
Plasmapheresis in hypertriglyceridemia-related pancreatitis: a case report
}

\author{
Andrea Tampieri, Patrizia Cenni, Claudia Morselli, Tiziano Lenzi \\ Emergency Department, S.M. Scaletta Hospital, Bologna
}

\section{Abstract}

Hypertriglyceridemia (HTG) is the third most common cause of acute pancreatitis (AP), accounting for up to $7 \%$ of cases. The clinical manifestations are similar to those of AP from other causes, but it may be difficult to recognize because of confounding laboratory investigations induced by HTG, such as a falsely normal serum amylase. Prompt recognition is important to provide adequate treatment. The maintenance of blood triglyceride (TG) levels below $500 \mathrm{mg} / \mathrm{dl}$ has been shown to accelerate the clinical improvement in patients with hypertriglyceridemic pancreatitis (HTGP). In many cases series apheresis was effective in reducing HTG and an early initiation is likely to be beneficial in order to prevent recurrence of AP and the development of necrotizing pancreatitis. Definitive guidelines for the treatment of HTGP and randomized trials that compare the effectiveness of apheresis with the medical therapy alone are still lacking.

\section{Case report}

A 50-year-old man was admitted to the Emergency Department referring severe epigastric pain and vomiting. Past medical history revealed recurrent abdominal pain, mild hyperlipidemia, poor compliance to lipid-lowering agents, and mild alcohol consumption, with no dietary excesses. Physical examination showed fever (body temperature $37,8^{\circ} \mathrm{C}$ ), normal vital signs (blood pressure 130/80; heart rate 95 beats/minute; oxygen saturation in ambience 96\%), and marked abdominal tenderness. Abdomen x-rays showed rare air-fluid ileal levels. Ultrasound examination excluded gallstones in the gallbladder and biliary tract, aortic diseases, and free fluid effusion in abdomen.

Laboratory routine test documented leukocytosis (white blood cells $15.100 \mathrm{u} / \mathrm{mmc}$ ) mild hyponatremia ( $123 \mathrm{mEq} / \mathrm{l}$; normal 134-148), mild hyperglycemia (171 mgldl), increased level of CPK (477 mu/ml; normal 0-200) and C-reactive protein (12.3 $\mathrm{mg} / \mathrm{dl}$; normal 0-0.5) without a significantly high level of serum amylase (104 mu/ml; normal 0-100). Haemoglobin, LDH, liver and renal function were normal, and the patient's serum sample was found to be lactescent.

An abdominal Computed Tomography Scan detected an interstitial pancreatitis with inflammatory changes in peri-pancreatic fatty tissue, multiple extra-pancreatic fluid collections with small pleural effusion (Balthazar CT severity index $=4$ ), and confirmed the absence of masses and biliary litiasis.

Second-line laboratory investigations showed increased levels of serum lipase up to $756 \mathrm{mu} / \mathrm{ml}$ (normal 13-60 ul), severe hypertriglyceridemia (> $5.000 \mathrm{mg} / \mathrm{dl}$; normal 50-170), high cholesterol serum level (1096 mg/dl; normal 150-240) with normal HDL. Thyroid function tests were normal.

Consistent with the diagnosis of hypertriglyceridemic pancreatitis (HTGP), the patient underwent a session of plasma exchange into referral center. Plasmapheresis was performed within 48 hours after admission, resulting in marked lowering of serum triglyceride (TG) levels $(678 \mathrm{mg} / \mathrm{dl})$.

Conventional treatment for acute pancreatitis (AP), including parenteral nutrition, aggressive hydration, intravenous ceftazidime administration, and analgesia were started contemporaneously.

Insulin was administered subcutaneously at the dose necessary to maintain blood glucose levels between $90-140 \mathrm{mg} / \mathrm{dl}$. Even so the laboratory tests worsened, showing a blood urea nitrogen rise of $22 \mathrm{mg} / \mathrm{dl}$, serum calcium 6,4 mg/dl, arterial $\mathrm{PO}_{2} 56 \mathrm{mmHg}$ and base deficit of 4,4 mmol/l, configuring a Ranson score 4 . Due to deteriorating conditions the patient was transferred to the intensive care unit to ensure an adequate fluid resuscitation, supplemental oxygen and careful monitoring. The patient's symptoms resolved over fourteen days, an ultrasound abdomen examination confirmed resolution of fluid collection, and serum lipase, electrolyte balance, glucose and PCR returned to normal levels. A specialist consultation with a metabolism physician was made and patient resulted affected by type IV dyslipidemia.

Oral anti-hyperlipidemic treatment with fenofibrate, rosuvastatin, and omega 3 fatty acids was stared as soon as possible; a dietary restriction of lipids and alcohol discontinuation was suggested before discharge at home. At the follow-up visit the patients was asymptomatic, and serum TG level, total cholesterol, LDL and HDL were normal.

\section{Discussion}

This case emphasizes the role of serum amylasemia as a pitfall in the diagnosis of AP; the relevance of HTG as an effectively treatable, metabolic cause of AP; the efficiency of apheresis in lowering serum TG levels and its possible usefulness in the management of HTGP. 


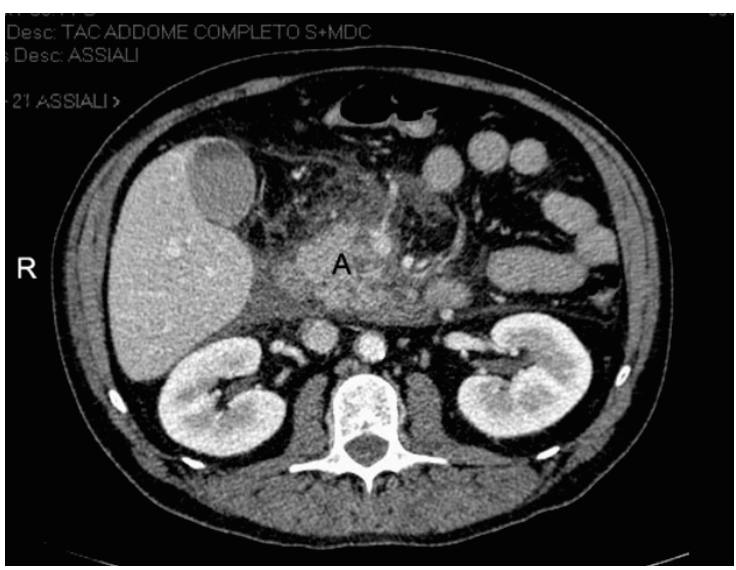

Fig. 1. R, right side; A, cephalic region of pancreas with interstitial pancreatitis, edema and fluid effusion in peri-pancreatic fatty tissue.

Fig. 2. R, right side; multiple extra-pancreatic fluid collections in right anterior para-renal space and latero-conal fascia (A), anterior renal fascia (B) and left latero-conal fascia (C).

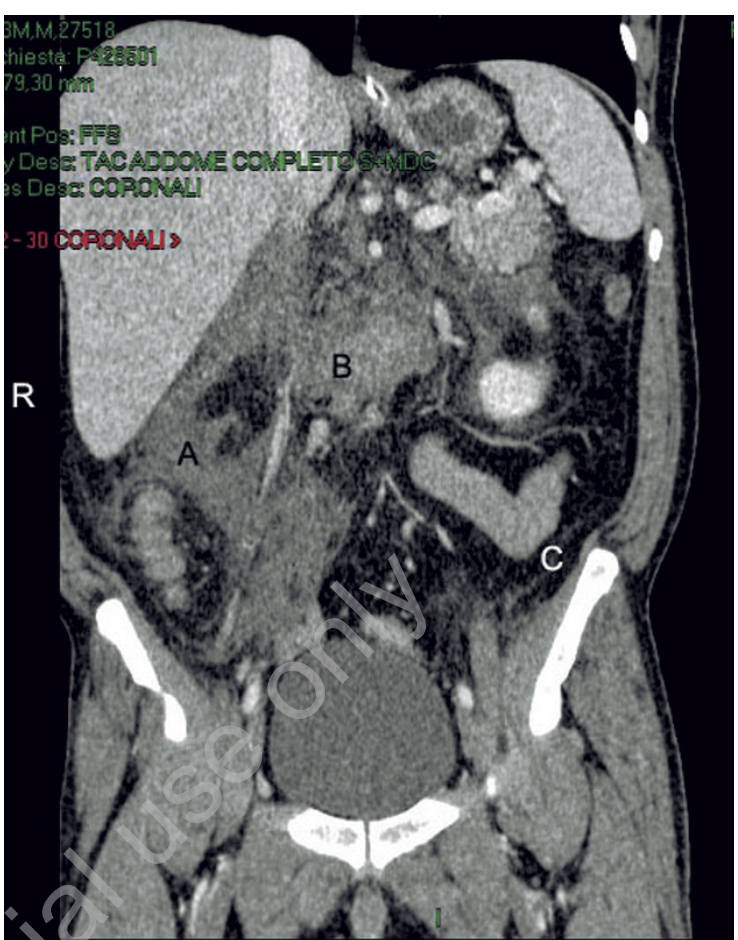

Acute abdominal pain is an extremely common cause of hospital visits, and often it represents a difficult challenge for the attending physician. Medical history and clinical features may make AP hard to distinguish from other conditions including perforated peptic ulcers, acute gastritis, biliary tract gallstones, or small bowel obstruction.

The laboratory tests most relied upon to substantiate the diagnosis of AP in ED are serum amylase and lipase, which are highly sensitive but not specific indexes, because increased levels also occur in other non pancreatic conditions.

The physician must be aware of possible confounding laboratory results due to elevated serum TG levels: a falsely normal amylasemia was reported when HTG level was more than $500 \mathrm{mg}$ ldl, while serum lipase confirmed an higher specificity and sensitivity for AP $(1,2)$. Moreover elevated TG levels can alter routine measurement of sodium causing a pseudo-hyponatremia, and lower the accuracy of Friedewald determination of LDL serum level $(3,4)$.

Even if chylomicronemia is not routinely investigated in ED, it should be considered as an unusual cause of acute abdominal pain with or without AP, and furthermore as a predisposing factor for mesenteric ischemia.

X-ray studies poorly contribute to the diagnosis of AP, and trans-abdominal ultrasounds show the pancreas in only $25-50 \%$ of patients with AP losing its value in demonstrating biliary tract disease.

Therefore a CT scan is recommended to confirm diagnosis especially when pancreatic enzymes are inconclusive or clinical findings suggest an alternative etiology of acute abdomen.

Actually the diagnostic criteria of AP require two of the following three features: a) characteristic abdominal pain, b) serum amylase and/or lipase more than three times the normal upper limit and c) findings of AP on CT scan, allowing for the eventuality of lower serum concentrations of pancreatic enzymes (5).

HTG is the third most common etiology of AP after gallstones and alcohol, accounting for 1-7\% of all cases (6-8), and it is reported to be responsible for up to $56 \%$ of AP cases during pregnancy (9).

Pathogenesis of HTGP is still unclear, but the most creditable theories suggest that free fatty acids, originated from the enzymatic degradation of excess TG, may produce pancreatic acinar cells and microvascular injury; besides hyperviscosity due to hyperchylomicronemia, impair circulation in pancreatic capillaries leading to ischemia.

Although clinical presentation is similar to AP due to other causes, recognition of poorly controlled diabetes, obesity, alcohol consumption, risk of drugs or diet-induced HTG, pregnancy, familiar history of hyperlipidemia, xanthomas, will suggest HTGP. A serum TG level of $1000 \mathrm{mgldl}$ or greater, or a recognized lactescent serum generated by chylomicrons formed in case of HTG even with triglyceridemia less than 1000 mgldl may induce HTGP, whereas TG levels less than 500mg\dl rule out HTGP (10).

Mild elevation of TG serum level is a well documented epiphenomenon in AP, but in this case it rapidly decreases after abstinence from eating, while persistent elevated levels reflect an underlying metabolic disorder. 
Both primary (genetic) and secondary causes of HTG should be evaluated after diagnosis of HTGP: Type IV dyslipidemia (Fredrickson classification) usually presents itself in adult-hood and requires another factor to raise serum TG levels and trigger AP; Type I and V can present HTGP without an exacerbating factor, and type I often occurs in infancy (11).

Various acquired conditions are responsible for a secondary HTG and can lead to HTGP.

Uncontrolled diabetes type 1 and 2, and ketoacidosis are the most common underlying causes of HTGP. Alcohol can induce HTGP, but it is still unclear if it directly increases serum TG concentrations or exacerbates a genetic HTG. Hypothyroidism has been documented as a cause of secondary HTG and implicated in HTGP (12).

Medications such as extrogen, tamoxifen, glucocorticoids, cyclosporine, protease inhibitors, isotretionin, propofol, olanzapine are involved in elevating level of serum TG.

Although pregnancy induced HTG has been found to be the major cause of gestational AP, the peak level of serum TG rarely exceeds $300 \mathrm{mg} / \mathrm{dl}$, suggesting that HTG may be attributable to other conditions or underlying familial HTG.

Severity and complications in HTGP have been reported higher than in AP from other causes, however mortality rates have not been found to differ and correlation between severity of HTGP and TG level has not been documented.

Despite its clinical relevance, official recommendations concerning specific management of HTGP are still lacking. In a recent review Tsuang et al. highlighted the role of apheresis in the treatment of HTGP and proposed an algorithm to approach and manage HTGP (13).

Initially the management of patients with AP is similar regardless of etiology, thus conventional treatment and clinical assessment should be started. The aggressive fluid replacement, total parenteral nutrition, antibiotic prophylaxis, analgesia and supportive care are the cornerstones of medical therapy $(5,14)$. Other potential causes of AP must be evaluated and treated (i.e. gallstones) as far as evaluation for primary or secondary causes of HTG must be performed when serum TG exceeds the level of $1000 \mathrm{mg} / \mathrm{dl}$. When the diagnosis of HTGP is confirmed, apheresis should be initiated within 48 hours if available and not contraindicated (i.e. in case of unstable vital signs).

This statement is recommended with a category 3 degree by the American society for apheresis guidelines 2007 consistent with limited data requiring further investigations (15).

The maintenance of blood TG levels below $500 \mathrm{mg} / \mathrm{dl}$ has been shown to accelerate the clinical improvement in patients with HTGP (7). Different studies have documented the efficiency of apheresis to reduce TG serum levels, and an early initiation of treatment is likely considered to be beneficial in patients with HTGP, although apheresis did not improve mortality and complications rates even in patients with severe disease defined as a Ranson's score greater than or equal to 3 points (16,17). It is well established that both insulin and heparin activate lipoprotein lipase consequently accelerate chylomicron degradation and effectively lower HTG, however the use of heparin in mono-therapy is controversial because it may lead to a depletion of lipoprotien lipase stores with a rebound effect. Intravenous administration of insulin titrated to maintain normal serum glucose level should be considered especially for the treatment of patients with HTGP and uncontrolled diabetes, being useful both to reduce HTG and hyperglycemia. Furthermore, insulin administration with subcutaneous or intravenous route has been shown to be effective in reducing TG serum levels in non-diabetic patients (18). Oral anti-hyperlipidemic agents should be initiated when tolerated.

\section{Conclusion}

Failure to consider HTG as a cause of AP may lead to misdiagnose the incidence of HTGP and lack appropriate management. An early initiation of apheresis rapidly reduces serum TG levels, removing the trigger that maintains the pancreatic damage. It is probably useful to accelerate the clinical improvement in patients with HTGP. However, a real improvement in mortality and complication rate has not been documented in small studies and case series. In this regard, larger randomized trials are needed to assess the true impact of apheresis in the management of HTGP.

\section{References}

1. Fallat RW, Vester JW et al. Suppression of amylase activity by hypertriglyceridemia. JAMA 1973; 225: 1331-4.

2. Tracy J, Wiliams A, Bais R et al. Evaluation of amylase and lipase in the diagnosis of acute pancreatitis. ANZ J Surg 2001; 71: 577-82.

3. Howard JM, Reed J. Pseudohyponatremia in acute hyperlipemic pancreatitis. A potential pitfall in therapy. Arch Surg 1985; 120: 1053-5.

4. Sniderman AD, Blank D et al. Triglycerides and small dense LDL: the twin Achilles heel of the Friedewald formula. Clin Biochem 2003; 36: 499-504.

5. Banks PA, Freeman ML et al. Practice guidelines in acute pancreatitis. Am J Gastroenterol 2006; 101: $2379-2400$.

6. Forston FR, Freedman SN, Webster PD III. Clinical assessment of hyperlipemic pancreatitis. Am J Gastroenterol 1995; 90: 2134-9.

7. Toskes PP. Hyperlipidemic pancreatitis. Gastroenterol Clin North Am 1990; 19: 783-91.

8. Dominguez-Munoz JE, Melfertheiner P et al. Hyperlipidemia in acute pancreatitis. Relationship with etiology, onset, and severity of the disease. Int J Pancreatol 1991; 10: 261-7. 


\section{casi clinici}

9. Chang CC, Hsieh YY, Tsai HD et al. Acute pancreatitis in pregnancy. Zhonghua Yi Xue Za Zhi 1998; 61: 85-92.

10. Yadav D, Pitchumoni SC. Issues in hyperilipidemic pancreatitis. J Clin Gastroenterol 2003; 36: 54-62.

11. Fredrickson DS. An international classification of hyperlipidemias and hyperlipoproteinemias. Ann Intern Med 1971; 75: 471-2.

12. Gan SI, Edwards AL et al. Hypertriglyceridemia-induced pancreatitis: a case-based review. World J Gastroenterol 2006; 12(44): 7197-202.

13. Tsuang W, Navaneethan U, Ruiz L et al. Hypertriglyceridemic pancreatitis: presentation and management. Am J Gastroenterol 2009; 104: 984-91.

14. GUT 2005.

15. Szczepiorkowsky ZM, Bandarenko N, Kim HC et al. Guidelines on the use of therapeutic apheresis in clinical practice. Evidence based approach from apheresis applications committee of the American society for apheresis. J Clin Apher 2007; 22: 106-75.

16. Kyriakidis AV, Raitsiou B et al. Management of acute severe hyperlipidemic pancreatitis. Digestion 2006; 73: $259-64$.

17. Chen JH, Yeh JH, Lai HW et al. Therapeutic plasma exchange in patients with hyperlipidemic pancreatitis. World J Gastroenterol 2004; 10: 2272-4.

18. Mikhail N, Trivedi K, Page C et al. Treatment of severe hypertriglyceridemia in nondiabetic patients with insulin. Am J Emerg Med 2005; 23: 415-7. 